\title{
Rodent Models to Study the Roles of the GH-IGF-1 Axis in the Aging and Anti-Aging Effects of Calorie Restriction
}

\author{
Ping Fang ${ }^{1,2}$, Maria L. Tagliaro ${ }^{1,3}$ and Isao Shimokawa ${ }^{*}, 1$ \\ ${ }^{I}$ Department of Investigative Pathology, Unit of Basic Medical Science, Graduate School of Biomedical Sciences, \\ Nagasaki University, Nagasaki, Japan \\ ${ }^{2}$ Department of Endocrinology and Metabolism, Shanghai Institute of Endocrine and Metabolic Diseases, Ruijin \\ Hospital, Shanghai Jiao Tong University School of Medicine, Shanghai 200025, People's Republic of China \\ ${ }^{3}$ Department of Morphophysiological Sciences, Faculty of Biosciences, Pontifical Catholic University of Rio Grande do \\ Sul (PUCRS), Porto Alegre, RS, Brazil
}

\begin{abstract}
Calorie restriction (CR) and inhibition of the growth hormone (GH)-insulin-like growth factor (IGF)-1 axis have been extensively investigated regarding their regulatory effects on aging processes and lifespan. Because CR moderately inhibits GH-IGF-1 signaling, the signal pathway may underlie the effect of CR. Our transgenic (tg/-) rat model, in which the GH-IGF-1 axis is modestly inhibited by overexpression of an antisense GH gene, showed similar characteristics to wild-type (WT)-CR rats in terms of lifespan extension, stress resistance and some neuroendocrine activities. Long-lived GH-receptor (GHR)-knockout (KO) mice, which are deficient in plasma IGF-1, showed no additional increase in lifespan by CR. These findings suggest importance of the GH signaling in the effect of CR. By contrast, the lifespan in Ames dwarf mice, in which the plasma IGF-1 level is also very low, was further increased by CR, suggesting presence of a pathway rather than the GH-IGF-1 axis in the effect of CR. Although the GH-IGF-1 axis may not be a sole pathway, many findings support the role for the GH-IGF-1 axis in the effects of CR. Longevity may be linked to retained cognitive functions in advanced ages. However, longevity models do not necessarily retain cognitive function or neuronal resistance to oxidative or neurotoxic stresses. IGF-1 signaling in the brain is differently regulated among those longevity models, even though systemic IGF-1 signaling is attenuated in most of the models. Knowledge on the regulation of the GH-IGF-1 axis in the brain in these longevity models could help us to devise interventions that retard agingdependent neurodegenerative processes.
\end{abstract}

Keywords: Calorie restriction, growth hormone, IGF-1, longevity, cognitive functions.

\section{INTRODUCTION}

Aging phenotypes are diverse, even in inbred strains of mice. Therefore, it seems unlikely that researchers could identify a fundamental mechanism(s) responsible for aging and thus devise anti-aging interventions. Surprisingly, however, a simple reduction in food or calorie intake, while providing essential nutrients, consistently retards many aging processes and even extends the lifespan in many organisms [1]. Spontaneous mutation or genetic manipulation of a single gene also extends lifespan in mammals as well as invertebrates [2]. These non-genetic and genetic longevity models have revealed evolutionary-conserved signal pathways that regulate aging and lifespan [3].

In rodents, over 20 genes have already been reported to promote longevity [2]. Many of these genes encode signaling molecules involved in energy metabolism and neuroendocrine systems. Of these, the growth hormone (GH)-

*Address correspondence to this author at the Department of Investigative Pathology, Unit of Basic Medical Science, Graduate School of Biomedical Sciences, Nagasaki University, Nagasaki, Japan; Tel: +81-95-819-7051; Fax:+81-95-819-7052; E-mail: shimo@nagasaki-u.ac.jp insulin-like growth factor (IGF)-1 axis is a particularly robust pathway that regulates lifespan in a range of organisms [2, 3]. Loss-of-function and reduction-of-function mutations in genes involved in this pathway prolong lifespan, as compared with their wild-type (WT) counterparts. In contrast, overexpression of the GH gene leads to premature aging and shortened lifespan [4]. In CR rodents, the plasma GH and/or IGF-1 levels are decreased [5-7]. Collectively, these findings indicate that the GH-IGF1 axis is a major signaling pathway that regulates the aging process and is thus involved in the anti-aging effects of CR.

In this chapter, we discuss the roles of the GH-IGF-1 axis in the regulation of aging and the effects of CR by summarizing recent findings in animal models of longevity. We pay particular attention to the similar neuroendocrine activities in transgenic dwarf rats and CR rats. Finally, we discuss findings on age-related cognitive function and neuronal resistance to stress in some of the longevity models, and the roles of the IGF-1 signaling pathway in the regulation of aging in the brain. 


\section{ROLES OF THE GH-IGF-1 AXIS IN THE EFFECTS OF CR}

The synthesis and secretion of GH in the pituitary gland are regulated by hypothalamic GH-releasing hormone (GHRH) and somatostatin (SRIH) in a competitive manner [8]. GH stimulates IGF-1 production, mostly in the liver. However, other tissues, such as the brain, skeletal muscle and white adipose tissue, also produce IGF-1 [10]. It is estimated that $80 \%$ of the circulating IGF- 1 is derived from the liver $[9,10]$; IGF-1 produced in the other tissues could also contribute to alterations in the plasma IGF-1 concentration to some extent.

Total or partial disruption of the hypothalamic-pituitaryperipheral tissue axis for GH and IGF-1 consistently extends the lifespan in rodents. For example, "little (lit/lit)" mice, in which the GHRH receptor (GHRH-R) gene is mutated, showed dwarfism and outlived WT mice when fed low protein diets [11]. KO of the $\mathrm{GH}$ receptor/binding protein (GHR) gene leads to GH resistance in peripheral tissues and thus plasma IGF-1 deficiencies, and ultimately extended the lifespan in mice [12]. Meanwhile, female, but not male, heterozygous IGF-1 receptor KO (IGF-1R (+/-)) mice also lived longer than WT mice [13]. Ames dwarf mice lacking pituitary $\mathrm{GH}$, prolactin (PRL) and thyroid-stimulating hormone (TSH) because of a loss-of-function mutation of the prop-1 gene also outlived their WT counterparts [14]. Although deficiencies in PRL and/or TSH might affect the lifespan of Ames mice, a recent study revealed that the GH signal plays a major role in lifespan extension in this model exhibiting pituitary dysfunction [15].

The GH-IGF-1 axis seems to be a key signaling pathway that mediates the effect of $\mathrm{CR}$, because $\mathrm{CR}$ abolishes the pulsatile secretion of GH at a young age [5], and modestly reduces the circulating IGF-1 levels in rodents $[6,7]$. However, epistasis analyses on Ames and GHR-KO mice are inconclusive. Both longevity models exhibit plasma IGF-1 deficiencies $[12,16]$, because of a deficit of GH secreting cells and GH resistance in peripheral tissues. Notably, CR did not further increase the lifespan in GHR-KO mice [17], but did further extend the lifespan in Ames mice [18]. The results obtained in GHR-KO mice emphasize the importance of GH-IGF-1 signaling in the effects of CR, while the findings in Ames mice suggest the presence of mechanisms other than GH-IGF-1 signaling.

In Ames mice, deficiencies in PRL and TSH might confound the effects of CR on longevity. For example, the mean lifespan of Ames mice fed ad libitum (AL) was reported to be 1000 days [14], while the lifespan of GHR-KO mice fed AL was 1139 days [12]. Although the genetic background of mice and animal husbandry differed between these studies, it seems that the deficits in PRL and TSH shortened the lifespan of Ames mice. In fact, experimentally induced hypothyroidism augments oxidative stress in rodents [19].

The genetic background of mice is also critical for the response to CR. In a study of 41 recombinant inbred strains of mice, $40 \%$ CR did not increase the lifespan in over $50 \%$ of the mouse strains [20]. In the study of GHR-KO mice, CR extended the lifespan of control wild-type mice (a cross of 129 Ola and Balbc mice) by $16 \%$ [17], while in the Ames mouse study, CR extended the lifespan of control WT mice (Oca2p/Oca2p prop1 df/+) by $32 \%$ [18]. Thus, the longer lifespan in the AL group of GHR-KO mice and the lower responsiveness of the genetic background of GHR-KO mice might mask the potential effects of $\mathrm{CR}$ in the GHR-KO mice, as compared with Ames mice.

\section{A TRANSGENIC DWARF RAT MODEL}

We previously reported that a strain of transgenic heterozygous ( $\mathrm{tg} /-$ ) rats, in which the pituitary GH synthesis and/or secretion is modestly inhibited by overexpression of antisense GH genes [21], outlived wild-type (WT) Wistar (Jcl:Wistar) rats, even if fed AL [22]. The plasma IGF-1 concentration in the tg/--AL rats was reduced by $60 \%$ of that in the WT-AL rats (Fig. 1A, Table 1; [7]). Meanwhile, the level in the WT-CR rats was reduced by $82 \%$ and $73 \%$ of that in the WT-AL rats in the fed and fasted phases of CR, respectively. Therefore, the GH-IGF-1 axis is modestly inhibited in the tg/- rats, as in WT-CR rats. CR in the tg/rats further decreased the plasma IGF-1 levels, even in the fed phase (Fig. 1A), indicating that the GH-IGF-1 axis in these rats is still responsive to reduced energy intake. Accordingly, the tg/- rat model differs from the GHR-KO and Ames dwarf mice, in which the plasma IGF-1 concentrations were extremely low $[12,16]$.

A series of experiments has revealed that the tg/--AL rats shared similarities with the WT-CR rats. The food intake of tg/--AL rats was $20-30 \%$ less than that of WT-AL rats during the lifespan study [22], and thus the food intake of $\mathrm{tg} /-$-AL rats was similar to that provided for the WT-CR $(30 \% \mathrm{CR})$ rats [7]. The body weight of tg/--AL rats was also similar to that of the WT-CR rats [7]. Non-fasting serum insulin levels [7] and glucose-stimulated serum insulin responses [23] were lower in tg/--AL and WT-CR rats than in the WT-AL rats. Stress resistance, which characterizes CR animals, was also noted in the tg/--AL rats [24]. These findings support the notion that modest inhibition of the GH-IGF-1 axis mediates some of the effects of CR.

We assessed the GHRH and SRIH-mRNA expression levels in the arcuate nuclei of hypothalamus in tg/- and WT rats fed AL or CR diets. The mRNA levels were measured by quantitative real-time PCR using mRNA extracted from microdissected arcuate nuclei, as described elsewhere [25, 26]. CR significantly reduced the GHRH-mRNA level in both tg/- and WT rats, regardless of the fed or fasted condition in the $\mathrm{CR}$ regimen (Fig. 1B). The tg/- rats exhibited lower levels of the GHRH-mRNA, although statistically insignificant. There was no significant difference in the SRIH-mRNA level between the WT and tg/- rats (Fig. 1C). CR did not significantly affect the SRIH-mRNA level.

As expected, the pituitary GH-mRNA expression level was significantly reduced in the tg/- rats (Fig. 1D). CR decreased the GH-mRNA expression level in WT rats, but not in tg/- rats. There was no significant difference between the CR-fed and fasted phases. Thus, in the WT rats, CR inhibits the stimulatory signal in the hypothalamus and in the pituitary gland. 

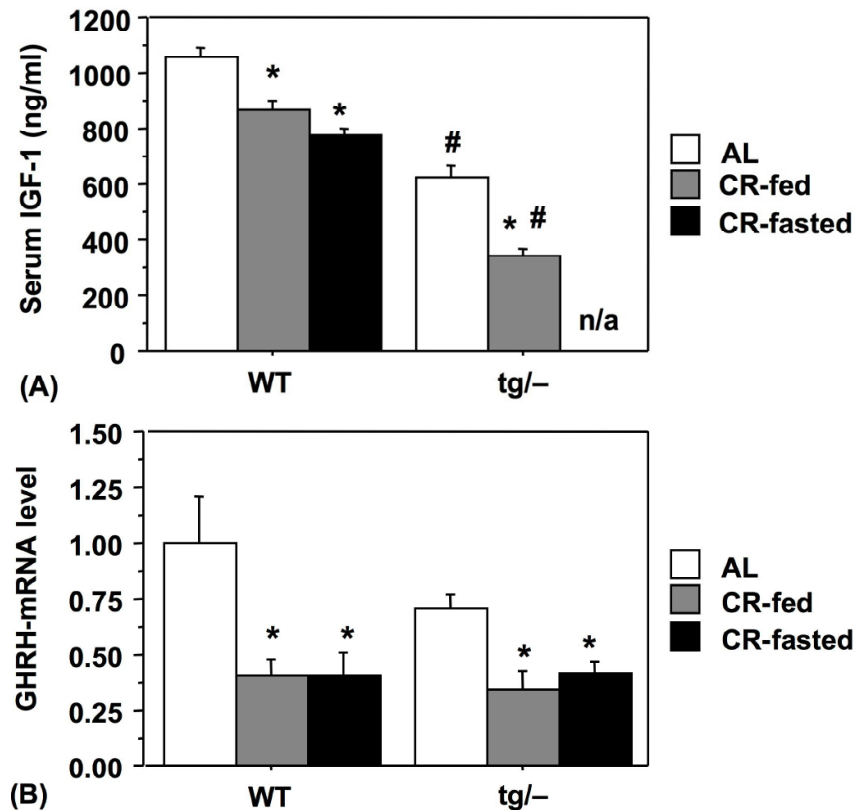

(B)
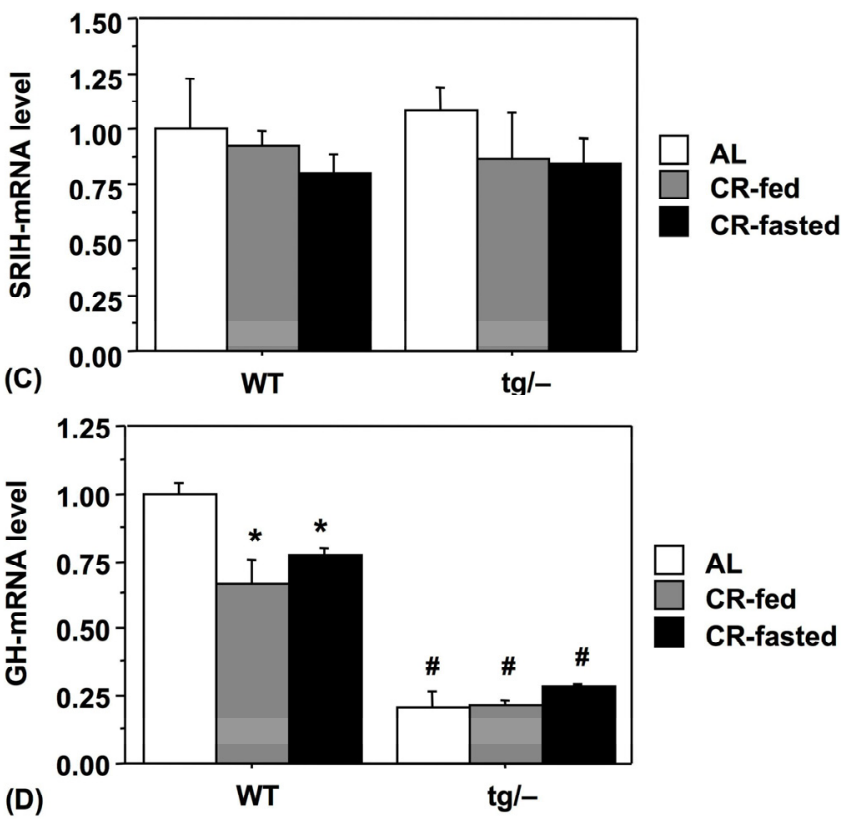

Fig. (1). The hypothalamic-pituitary axis in wild-type (WT) calorierestricted (CR) rats and transgenic (tg/-) fed ad libitum (AL) rats in a feeding cycle at 6-months of age. A) Plasma IGF-1 concentrations. B) Growth hormone (GH)-releasing hormone (GHRH) and C) somatostatin (SRIH) mRNA expression in the arcuate nucleus of the hypothalamus. D) Pituitary GH-mRNA expression. Bars represent mean \pm standard error $(n=5-12$ for plasma IGF-1; $\mathrm{n}=5-6$ for GHRH and SRIH mRNA; $\mathrm{n}=3-4$ for pituitary GH mRNA). * $\mathrm{p}<0.05$ versus the corresponding $\mathrm{AL}$ groups and $\# \mathrm{p}<0.05$ versus each feeding phase in the WT rat group by one-factor ANOVA followed by the Tukey-Kramer post hoc test for multiple comparisons. The materials and methods are published in more detail elsewhere [25, 28].

In tg/- rats, the GH-mRNA expression was not significantly different between the feeding phases. The anti$\mathrm{GH}$ transgene, which consisted of four copies of the thyroid hormone response element, the rat $\mathrm{GH}$ promoter, and antisense cDNA sequences for rat $\mathrm{GH}$, was genetically engineered to be expressed in a similar manner to that of the intrinsic GH gene [21]. The expression of anti-GH-mRNA was confirmed in the expected tissues, namely the pituitary gland and the thymus, but not in the other tissues examined [22]. These findings on GH-mRNA in tg/--AL rats indicate that the antisense GH-mRNA effectively antagonizes $\mathrm{GH}$ mRNA expression.

Our studies also showed that the expression of antisense GH-mRNA from early development modestly inhibited the somatotrophic axis and lead to smaller body size in Wistar male rats. The tg/- rats responded to $30 \% \mathrm{CR}$ by further suppression of the somatotrophic axis and lifespan extension [7].

Circulating IGF-1 inhibits the pituitary gland and the hypothalamus by negative feedback pathways [8]. Under physiological conditions but not extreme conditions (e.g., protein malnutrition or long-term fasting), the hypothalamicpituitary-peripheral tissue axis maintains somatotrophic homeostasis via this feedback mechanism [27].

We evaluated the feedback response to recombinant human (rh)IGF-1 in WT-AL, WT-CR and tg/--AL rats [28]. rhIGF-1 or control saline was administered for 14 days via osmotic mini-pumps that were implanted subcutaneously in the interscapular region. rhIGF-1 administration increased body weight in all three groups of rats by about $3 \%$ increase, but it did not affect cumulative food intake [28]. The mass of the perirenal fat pad, normalized for body weight, was decreased by $23 \%$ in WT-AL rats, but was unchanged in the WT-CR and tg/--AL rats. The metabolic actions of IGF-1 are complex, exerting GH-like and insulin-like effects in vivo depending on the dose and the mode of administration [29]. In most cases, IGF-1 mediates the protein anabolic actions of $\mathrm{GH}$ by selectively increasing whole-body protein synthesis [30]. GH also exhibits a lipolytic effect that is independent of IGF-1 and is probably achieved through changes in insulin levels and activity [31].

Administration of rhIGF-1 for 14 days activated the endogenous negative feedback pathway controlling the GHIGF-1 axis. As a result, the pituitary GH-mRNA levels were slightly reduced by rhIGF-1 (Fig. 2A, Table 2), with a greater effect in WT-AL rats than in tg/-AL rats. Of the total serum IGF-1, 20-30\% was replaced by human IGF-1 (Fig. 2B), and reduced the rat native IGF-1 levels by an equivalent amount.

In the rhIGF-1 infusion experiment, we determined the mRNA levels in the liver and white adipose tissue. In the liver, the IGF-1-mRNA level did not differ between the WTAL and WT-CR rats (Fig. 2C), but was lower in the Tg-AL rats. rhIGF-1 infusion did not significantly affect IGF-1mRNA expression. In perirenal fat tissue, IGF-1-mRNA expression was greatest in the WT-AL rats (Fig. 2D), but was not different between the WT-CR and Tg-AL rats. Notably, rhIGF-1 infusion significantly reduced the mRNA level only in the perirenal fat tissue of the WT-AL rats.

Hepatic IGF-1- mRNA expression does not seem to be directly regulated by IGF-1 in adult rats, because there are very few IGF-1 binding sites in the adult rat liver [32]. In contrast, conditional IGF-1R inactivation in adipose tissue 

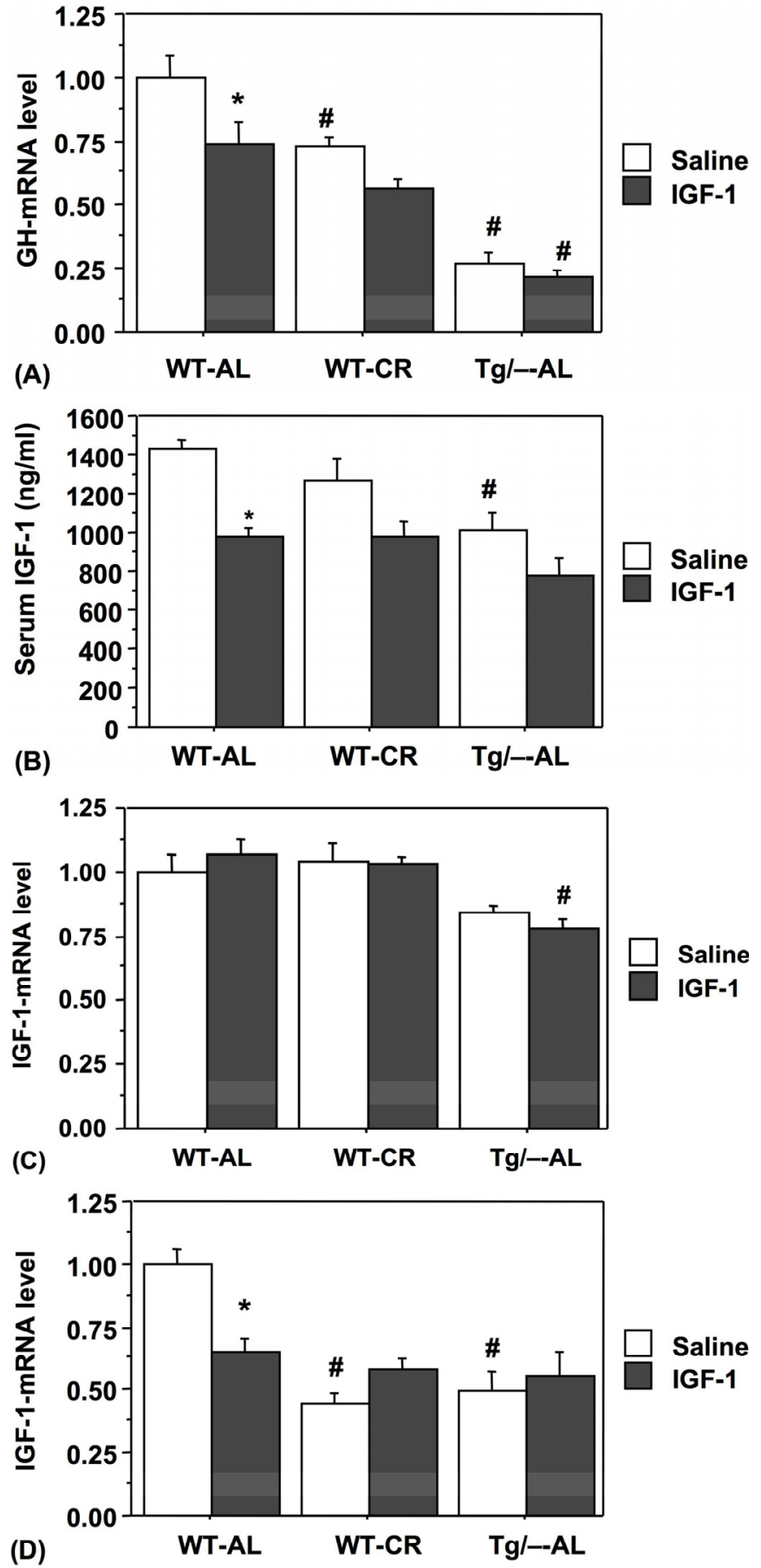

Fig. (2). Effects of recombinant human (rh) insulin-like growth factor (IGF)-1 administration on the pituitary-peripheral tissue axis in wild-type (WT) calorie-restricted (CR) rats and transgenic (tg/-) rats fed ad libitum (AL). A) Serum IGF-1 concentration. B) Pituitary GH-mRNA expression. $\mathrm{C}$ and D) IGF-1-mRNA expression in the liver (C) and perirenal adipose tissue (D). The details are described elsewhere [28]. It should be noted that the experimental rats were killed at 9 months of age after an overnight fast, which decreased the difference in serum IGF-1 levels between the WT-AL and WT-CR rats. Of the data published [28], only the saline-injected groups were used to develop these figures. Bars represent mean \pm standard error $(n=4-5)$. $* p<0.05$ versus the corresponding AL groups and $\# \mathrm{p}<0.05$ versus each feeding phase in the WT rat group by one-factor ANOVA followed by the TukeyKramer post hoc test for multiple comparisons. increased somatic growth by elevating serum IGF-1 concentrations in mice [33]. In these mice, the IGF-1-mRNA levels were increased in adipose tissue and in the liver. Those findings suggest that the IGF-1 signal in adipose tissue participates in somatotrophic homeostasis.

In our study, the hepatic IGF-1-mRNA was not significantly affected by rhIGF-1 infusion in all three rat groups. However, adipose tissue IGF-1-mRNA expression was considerably reduced by rhIGF-1, but only in the WTAL rats. The decreased serum IGF-1 concentration in the WT-AL rats could be explained by the reduced IGF-1mRNA expression in the adipose tissue, and by the fact that the size of the fat pad was significantly decreased in the WT$\mathrm{AL}$ rats [28]. The adipose tissue in the WT-CR and Tg-AL rats was refractory to exogenously administered rhIGF-1 in terms of IGF-1-mRNA expression. Nonetheless, the serum native IGF-1 level decreased in the WT-CR and Tg-AL rats in a similar fashion to that in the WT-AL rats. These findings suggest that $\mathrm{CR}$ causes specific changes in the regulation of serum levels of IGF-1, and that modest inhibition of the GH axis mimics the CR-specific metabolic milieu that is associated with the adipose tissue. Adiponectin, which is secreted from adipose tissue, sensitizes insulin actions and attenuates inflammation in vessels [34, 35]. Interestingly, overexpression of adiponectin was reported to prolong the lifespan in mice [36]. rhIGF-1 infusion reversed the increased plasma adiponectin concentration in WT-CR and $\mathrm{tg}$ /--AL rats to a level similar to that in WT-AL rats [28]. Thus, metabolic changes in the adipose tissue induced by modest inhibition of the GH-IGF-1 axis could be attributable to the anti-aging effects of $\mathrm{CR}$, although the precise mechanisms involved are elusive. Collectively, our studies suggest that the GH-IGF-1 axis is a key signaling pathway in the effects of CR.

\section{COGNITIVE FUNCTIONS AND NEURONAL RESISTANCE TO STRESS IN THE LONGEVITY MODELS}

Longevity may be linked to the retention of cognitive functions in older age. In other words, a mechanism by which lifespan, particularly a healthy lifespan, is prolonged also protects against the onset of age-related cognitive disorders.

There is ample evidence showing the beneficial effects of $\mathrm{CR}$ on cognitive functions in aging rodents. However, many of these findings have been derived from disease models, including high-fat diet models and Alzheimer disease models $[37,38]$. The effects of CR on age-related cognitive disorders in rodents fed standard diets are controversial. A behavioral test (the Barnes maze test) in a mouse model of Alzheimer disease showed that $\mathrm{CR}$ enhanced the learning of new tasks and the retention of the learned tasks in the model mice [39]. However, in the control WT mice, CR did not significantly affect spatial reference memory performance. Yanai et al. [40] also reported that $\mathrm{CR}$ had negative effects on cognitive function in rats, although the level of dietary restriction seemed to be more severe than the standard experimental levels (30-40\%), as the body weight was only $280 \mathrm{~g}$ in the CR group versus approximately $800 \mathrm{~g}$ in the $\mathrm{AL}$ group. A recent 
Table 1. The Hypothalamic-Pituitary Axis in Wild-Type (WT) Calorie-Restricted (CR) Rats and Transgenic (tg/-) Fed ad Libitum (AL) Rats in a Feeding Cycle at 6-Months of Age

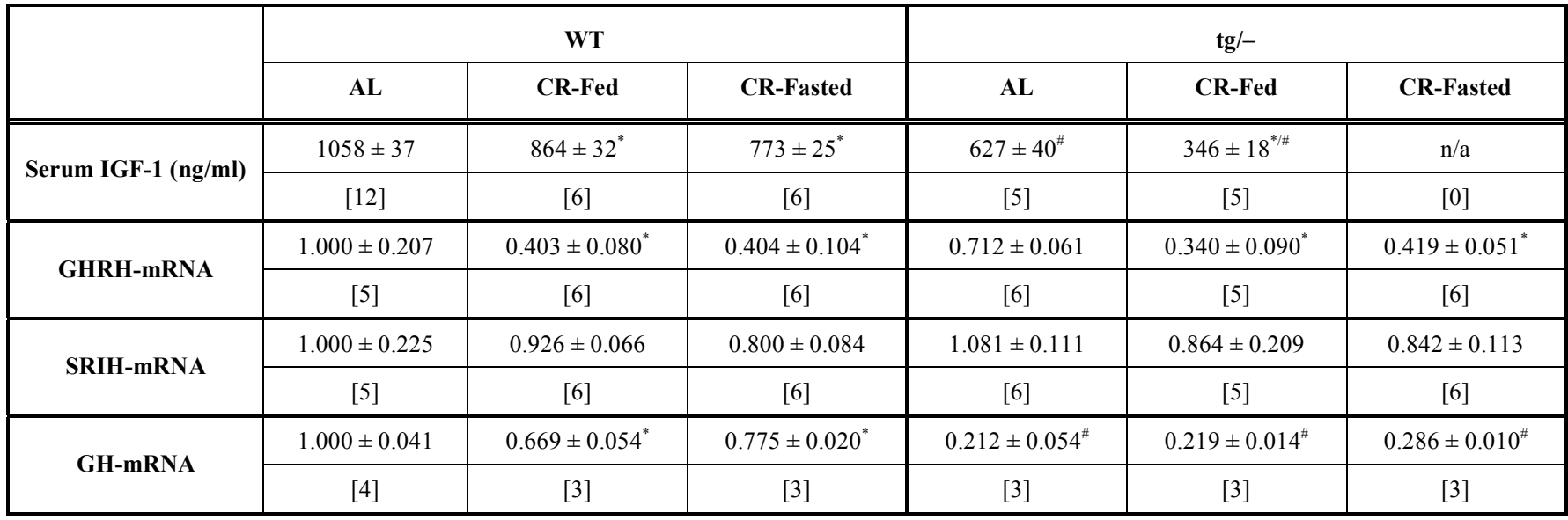

Data are illustrated in Fig. (1). Data represent means \pm SE [number of rats examined]. The mRNA expression levels are normalized by those in the WT-AL group. GHRH, growth hormone-releasing hormone: SRIH, somatostatin: GH, growth hormone: CR-fed and CR-fasted, $30 \%$ dietary restricted feeding groups while sacrificed in the first day and the second day of 2-day feeding cycle respectively (see the rat husbandry for the CR regimen [26]). ${ }^{*} \mathrm{p}<0.05$ versus the corresponding AL groups and \# $\mathrm{p}<0.05$ versus each feeding phase in the WT rat group by one-factor ANOVA followed by the Tukey-Kramer post hoc test for multiple comparisons.

Table 2. Effects of Recombinant Human (rh) Insulin-Like Growth Factor (IGF)-1 Administration on the Pituitary-Peripheral Tissue Axis in Wild-Type (WT) Calorie-Restricted (CR) Rats and Transgenic (tg/-) Rats Fed ad Libitum (AL)

\begin{tabular}{|c|c|c|c|c|c|c|}
\hline \multirow{2}{*}{} & \multicolumn{2}{|c|}{ WT-AL } & \multicolumn{2}{|c|}{ WT-CR } & \multicolumn{2}{|c|}{ Tg/- } \\
\cline { 2 - 7 } & Saline & IGF-1 & Saline & IGF-1 & Saline & IGF-1 \\
\hline \hline \multirow{2}{*}{ GH-mRNA } & $1.000 \pm 0.088$ & $1.157 \pm 0.098^{*}$ & $1.134 \pm 0.077^{\#}$ & $1.031 \pm 0.028$ & $0.842 \pm 0.026^{\#}$ & $0.781 \pm 0.036^{\#}$ \\
\cline { 2 - 7 } & {$[5]$} & {$[5]$} & {$[5]$} & {$[5]$} & {$[4]$} & {$[5]$} \\
\hline \multirow{2}{*}{ Serum IGF-1 (ng/ml) } & $1433 \pm 50$ & $975 \pm 47^{*}$ & $1269 \pm 113$ & $975 \pm 77$ & $1014 \pm 90^{\#}$ & $779 \pm 90$ \\
\cline { 2 - 7 } & {$[5]$} & {$[5]$} & {$[5]$} & {$[5]$} & {$[4]$} & {$[5]$} \\
\hline \multirow{2}{*}{ Hepatic IGF-1 mRNA } & $1.000 \pm 0.068$ & $1.157 \pm 0.098$ & $1.134 \pm 0.077$ & $1.031 \pm 0.028$ & $0.842 \pm 0.026$ & $0.781 \pm 0.036^{\#}$ \\
\cline { 2 - 7 } & {$[5]$} & {$[5]$} & {$[5]$} & {$[5]$} & {$[4]$} & {$[5]$} \\
\hline \multirow{2}{*}{ Perirenal fat IGF-1 mRNA } & $1.000 \pm 0.213$ & $0.486 \pm 0.059^{*}$ & $0.202 \pm 0.068^{\#}$ & $0.198 \pm 0.025$ & $0.292 \pm 0.057^{\#}$ & $0.365 \pm 0.119$ \\
\cline { 2 - 7 } & {$[5]$} & {$[5]$} & {$[5]$} & {$[5]$} & {$[4]$} & {$[5]$} \\
\hline
\end{tabular}

Data are illustrated in Fig. (2). Data represent means \pm SE [number of rats examined]. The mRNA expression levels are normalized by those in the WT-AL group. The details are described elsewhere [28]. $* \mathrm{p}<0.05$ versus the corresponding AL groups and \# $\mathrm{p}<0.05$ versus each feeding phase in the WT rat group by one-factor ANOVA followed by the Tukey-Kramer post hoc test for multiple comparisons.

study also showed that CR resulted in an overall increase in physical activity, which may confound the outcomes of behavioral cognitive function tests [41]. Thus, rodent experiments to examine the effects of $\mathrm{CR}$ on cognitive function should be carefully conducted and interpreted.

Snell mice, which show the same phenotype to that of Ames mice and also live longer [11], have been reported to exhibit abnormal behaviors and a reduction in the number of hypothalamic neurons [42]; recent studies have shown that Ames mice retain their cognitive function and locomotor activities to advanced ages [43]. In accordance with these findings, Ames mice had greater numbers of new neuronal cells in the granular cell layer of the dentate gyrus of the hippocampus at 3 and 20 months of age [44, 45]. In the hippocampus, IGF-1 protein and phosphorylated Akt levels were also increased, suggesting that the IGF-1-mediating cell survival signal is augmented in Ames mice as compared to wild type mice. Concomitantly, the expression level of an anti-apoptotic protein (Bcl-2) was greater in Ames mice, whereas $\mathrm{Bad}$, an apoptotic protein, was predominantly phosphorylated and thus inactivated, and cleaved caspase-9, a proapoptotic protein, was decreased in Ames mice [45]. Collectively, these findings suggest that hippocampal neurons are relatively preserved by the enhanced IGF-1 signaling in Ames mice, thus maintaining cognitive function to an advanced age.

Using an organotypic slice system of neurons cultured from hippocampi of adult Ames mice and WT mice, Schrag et al. [46] reported that the hippocampal slices from Ames mice were resistant to beta-amyloid-induced hyperphosphorylation of Tau protein, which promotes neuronal death. In contrast, another study showed that Ames dwarf mice are more vulnerable to kainic acid (KA), which is neuroexcitotoxic and epileptogenic acting through specific kainite ionotropic receptors [47]. In that study, administration of KA induced seizure and hippocampal 
neurodegeneration in Ames mice, but not in WT type mice. In fact, a 2-fold higher dose of KA was required in WT mice to induce similar degrees of seizure and neurodegeneration. The hippocampal-based spatial memory of Ames and WT mice, in which a similar degree of neurodegeneration was induced by different doses of KA (equiseizure dose), was evaluated by the Barnes maze and the T-maze. Intriguingly, in that study, Ames mice retained their spatial memory as compared with the WT mice [47]. Sharma and colleagues concluded that Ames dwarf mice had an enhanced defense capacity against the neurotoxin in the hippocampus, although the interpretation seems to be controversial.

In contrast to Ames mice, IGF-1R (+/-) mice are more vulnerable to 1-methyl-4-phenyl-1,2,3,6-tetrahydropyridine (MPTP), a neurotoxin precursor metabolized into the toxic cation 1-methyl-4-phenylpyridinium by monoamine oxidase B [48]. Indeed, MPTP induced more severe lesions in the dopaminergic neurons of the substantia nigra in IGF-1R (+/) mice than in WT mice. In this situation, the ROS and NO levels were lower in IGF-1R (+/-) mice, suggesting that the vulnerability is not caused by oxidative stress. Microarray analysis suggested an antineuroinflammatory role of IGF-1, as its the gene expression profile reflected an exaggerated inflammatory reaction that may explain the increased damage in the brain of IGF-1R (+/-) mice [48]. It should be noted, however, that this experiment was conducted in male $\mathrm{KO}$ mice that did not exhibit significantly increased lifespan or oxidative stress resistance [13]. In the original report, only female IGF-1R (+/-) mice had a prolonged lifespan and resistance to oxidative stress.

Replenishment of $\mathrm{GH}$ in Lewis strain-derived dwarf $(\mathrm{dw} / \mathrm{dw})$ rats aged between 4 and 15 weeks old increased their lifespan when compared with control $(\mathrm{dw} /+)$ and untreated $\mathrm{dw} / \mathrm{dw}$ rats [49], although the life-prolonging effect was observed in male but not female rats. Since the plasma IGF-1 returned to the baseline level (40\% lower than the level in control non-dwarf rats) after stopping $\mathrm{GH}$ treatment, the paradigm was designated as adult-onset $\mathrm{GH}$ and IGF-1 deficiency (AO-GHD). In the AO-GHD model, IGF-1 concentrations in the cerebrospinal fluid (CSF) were also increased during the $\mathrm{GH}$ administration, whereas the CSF IGF-1 level declined to the baseline levels in the dwarf rats after stopping $\mathrm{GH}$ treatment [50]. In this AO-GHD model, the cell proliferation rate in the DG in the hippocampus did not differ from those in GH-replenished dwarf rats and control dwarf rats. GH-IGF-1 deficiency did not affect the differentiation of new cells into neuronal cells. However, the number of new neurons in the DG was reduced in the AO-GHD rats. These findings also suggest the prosurvival effects of the local IGF-1 signal in neuronal cells. GH treatment increases glucose utilization and energy metabolism in the brain [51], while deficiencies in circulating $\mathrm{GH} / \mathrm{IGF}-1$ potentially promote brain aging.

In summary, reduced GH-IGF-1 signaling prolongs the lifespan of rodents. The mechanism is probably involved in the effect of CR. However, these longevity paradigms are not consistently linked to the preservation of cognitive function, neuronal resistance to stress, or the survival of neurons in the brain. Although the plasma IGF-1 level was reduced or IGF-1R was inactivated in the target tissues in the longevity models, only Ames dwarf mice show higher levels of IGF-1 in the brain. The molecular basis of the regulation of IGF-1 signaling in peripheral tissues, particularly in the brain, should be investigated in these animal models of longevity.

\section{CONFLICT OF INTEREST}

The authors confirm that this article content has no conflicts of interest.

\section{ACKNOWLEDGEMENTS}

This work was supported by the Grant-in-Aid for Scientific research from Japan Society fro Promotion of Science (JSPS, no. 12680779) and also, in part, by the Asian CORE program of JSPS.

\section{REFERENCES}

[1] Masoro EJ. Subfield history: caloric restriction, slowing aging, and extending life. Sci Aging Knowledge Environ 2003; 2003: RE2.

[2] Shimokawa I, Chiba T, Yamaza H, Komatsu T. Longevity genes: insights from calorie restriction and genetic longevity models. Mol Cells 2008; 26: 427-35.

[3] Longo VD, Finch CE. Evolutionary medicine: from dwarf model systems to healthy centenarians? Science 2003; 299: 1342-6.

[4] Steger RW, Bartke A, Cecim M. Premature ageing in transgenic mice expressing different growth hormone genes. J Reprod Fertil Suppl 1993; 46: 61-75.

[5] Sonntag WE, Steger RW, Forman LJ, Meites J. Decreased pulsatile release of growth hormone in old male rats. Endocrinology 1980; 107: $1875-9$

[6] Breese CR, Ingram RL, Sonntag WE. Influence of age and longterm dietary restriction on plasma insulin-like growth factor-1 (IGF-1), IGF-1 gene expression, and IGF-1 binding proteins. J Gerontol 1991; 46: B180-7.

[7] Shimokawa I, Higami Y, Tsuchiya T, et al. Life span extension by reduction of the growth hormone-insulin-like growth factor-1 axis: relation to caloric restriction. FASEB J 2003; 17: 1108-9.

[8] Herman-Bonert V, Prager D, Melmed S. In: Melmed S, Ed. The Pituitary. Cambridge: Blackwell Science, Inc. 1995; pp. 98-135.

[9] Sjögren K, Jansson J-O, Isaksson OGP, Ohlsson C. A transgenic model to determine the physiological role of liver-derived insulinlike growth factor I. Minerva Endocrinol 2002; 27: 299-311.

[10] Yakar S, Liu JL, Stannard B, et al. Normal growth and development in the absence of hepatic insulin-like growth factor I. Proc Natl Acad Sci USA 1999; 96: 7324-9.

[11] Flurkey K, Papaconstantinou J, Miller RA, Harrison DE. Lifespan extension and delayed immune and collagen aging in mutant mice with defects in growth hormone production. Proc Natl Acad Sci USA 2001; 98: 6736-41.

[12] Coschigano KT, Clemmons D, Bellush LL, Kopchick JJ. Assessment of growth parameters and life span of GHR/BP genedisrupted mice. Endocrinology 2000; 141: 2608-13.

[13] Holzenberger M, Dupont J, Ducos B, et al. IGF-1 receptor regulates lifespan and resistance to oxidative stress in mice. Nature 2003; 421: 182-7.

[14] Brown-Borg HM, Borg KE, Meliska CJ, Bartke A. Dwarf mice and the ageing process. Nature 1996; 384: 33 .

[15] Panici JA, Harper JM, Miller RA, et al. Early life growth hormone treatment shortens longevity and decreases cellular stress resistance in long-lived mutant mice. FASEB J 2010; 24: 5073-9.

[16] Chandrashekar V, Bartke A. Induction of endogenous insulin-like growth factor-I secretion alters the hypothalamic-pituitarytesticular function in growth hormone-deficient adult dwarf mice. Biol Reprod 1993; 48: 544-51.

[17] Bonkowski MS, Rocha JS, Masternak MM, Al Regaiey KA, Bartke A. Targeted disruption of growth hormone receptor interferes with the beneficial actions of calorie restriction. Proc Natl Acad Sci USA 2006; 103: 7901-5. 
[18] Bartke A, Wright JC, Mattison JA, et al. Extending the lifespan of long-lived mice. Nature 2001; 414: 412.

[19] Yilmaz S, Ozan S, Benzer F, Canatan H. Oxidative damage and antioxidant enzyme activities in experimental hypothyroidism. Cell Biochem Funct 2003; 21: 325-30.

[20] Liao CY, Rikke BA, Johnson TE, Diaz V, Nelson JF. Genetic variation in the murine lifespan response to dietary restriction: from life extension to life shortening. Aging Cell 2010; 9: 92-5.

[21] Matsumoto K, Kakidani H, Takahashi A, et al. Growth retardation in rats whose growth hormone gene expression was suppressed by antisense RNA transgene. Mol Reprod Dev 1993; 36: 53-8.

[22] Shimokawa I, Higami Y, Utsuyama M, et al. Life span extension by reduction in growth hormone-insulin-like growth factor-1 axis in a transgenic rat model. Am J Pathol 2002; 160: 2259-65.

[23] Yamaza H, Komatsu T, Chiba T, et al. A transgenic dwarf rat model as a tool for the study of calorie restriction and aging. Exp Gerontol 2004; 39: 269-72.

[24] Komatsu T, Trindade L, Chiba T, et al. Acute stress response modified by modest inhibition of growth hormone axis: a potential machinery of the anti-aging effect of calorie restriction. Mech Ageing Dev 2011: 132(3): 103-9.

[25] Komatsu T, Chiba T, Yamaza H, et al. Effect of leptin on hypothalamic gene expression in calorie-restricted rats. J Gerontol A Biol Sci Med Sci 2006; 61: 890-8.

[26] Shimokawa I, Fukuyama T, Yanagihara-Outa K, et al. Effects of caloric restriction on gene expression in the arcuate nucleus. Neurobiol Aging 2003; 24: 117-23.

[27] Scacchi M, Ida Pincelli A, Cavagnini F. Nutritional status in the neuroendocrine control of growth hormone secretion: the model of anorexia nervosa. Front Neuroendocrinol 2003; 24: 200-24.

[28] Yamaza H, Komatsu T, To K, et al. Involvement of insulin-like growth factor-1 in the effect of caloric restriction: regulation of plasma adiponectin and leptin. J Gerontol A Biol Sci Med Sci 2007; 62: 27-33.

[29] Mauras N, Haymond MW. Metabolic effects of recombinant human insulin-like growth factor-I in humans: comparison with recombinant human growth hormone. Pediatr Nephrol 1996; 10: 318-23.

[30] Mauras N, Martinez V, Rini A, Guevara-Aguirre J. Recombinant human insulin-like growth factor I has significant anabolic effects in adults with growth hormone receptor deficiency: studies on protein, glucose, and lipid metabolism. J Clin Endocrinol Metab 2000; 85: 3036-42.

[31] Hussain MA, Schmitz O, Mengel A, et al. Comparison of the effects of growth hormone and insulin-like growth factor I on substrate oxidation and on insulin sensitivity in growth hormonedeficient humans. J Clin Invest 1994; 94: 1126-33.

[32] Caro JF, Poulos J, Ittoop O, et al. Insulin-like growth factor I binding in hepatocytes from human liver, human hepatoma, and normal, regenerating, and fetal rat liver. J Clin Invest 1988; 81: 976-81.

[33] Klöting N, Koch L, Wunderlich T, et al. Autocrine IGF-1 action in adipocytes controls systemic IGF-1 concentrations and growth. Diabetes 2008; 57: 2074-82.

[34] Fasshauer M Paschke R. Regulation of adipocytokines and insulin resistance. Diabetologia 2003; 46: 1594-603.

[35] Havel PJ. Update on adipocyte hormones: regulation of energy balance and carbohydrate/lipid metabolism. Diabetes 2004; 53 (Suppl 1): S143-51.
[36] Otabe S, Yuan X, Fukutani T, et al. Overexpression of human adiponectin in transgenic mice results in suppression of fat accumulation and prevention of premature death by high-calorie diet. Am J Physiol Endocrinol Metab 2007; 293: E210-8.

[37] Komatsu T, Chiba T, Yamaza H, et al. Manipulation of caloric content but not diet composition, attenuates the deficit in learning and memory of senescence-accelerated mouse strain P8. Exp Gerontol 2008; 43: 339-46.

[38] Mattson MP. The impact of dietary energy intake on cognitive aging. Front Aging Neurosci 2010; 2: 5 .

[39] Qin W, Zhao W, Ho L, et al. Regulation of forkhead transcription factor FoxO3a contributes to calorie restriction-induced prevention of Alzheimer's disease-type amyloid neuropathology and spatial memory deterioration. Ann N Y Acad Sci 2008; 1147: 335-47.

[40] Yanai S, Okaichi Y Okaichi H. Long-term dietary restriction causes negative effects on cognitive functions in rats. Neurobiol Aging 2004; 25: 325-32.

[41] Carter CS, Leeuwenburgh C, Daniels M, Foster TC. Influence of calorie restriction on measures of age-related cognitive decline: role of increased physical activity. J Gerontol A Biol Sci Med Sci 2009; 64: 850-9.

[42] Kempf E, Fuhrmann G, Thiriet G, Ebel A. Dopamine and serotonin metabolism in striatum and in the septohippocampal pathway of the Snell dwarf mouse. Neurochem Res 1985; 10: 969-81.

[43] Kinney BA, Meliska CJ, Steger RW, Bartke A. Evidence that Ames dwarf mice age differently from their normal siblings in behavioral and learning and memory parameters. Horm Behav 2001; 39: 277-84.

[44] Sun LY, Evans MS, Hsieh J, Panici J, Bartke A. Increased neurogenesis in dentate gyrus of long-lived Ames dwarf mice. Endocrinology 2005; 146: 1138-44.

[45] Sun LY, Bartke A. Adult neurogenesis in the hippocampus of longlived mice during aging. J Gerontol A Biol Sci Med Sci 2007; 62: 117-25.

[46] Schrag M, Sharma S, Brown-Borg H Ghribi O. Hippocampus of Ames dwarf mice is resistant to beta-amyloid-induced tau hyperphosphorylation and changes in apoptosis-regulatory protein levels. Hippocampus 2008; 18: 239-44.

[47] Sharma S, Haselton J, Rakoczy S, Branshaw S, Brown-Borg HM. Spatial memory is enhanced in long-living Ames dwarf mice and maintained following kainic acid induced neurodegeneration. Mech Ageing Dev 2010; 131: 422-35.

[48] Nadjar A, Berton O, Guo S, et al. IGF-1 signaling reduces neuroinflammatory response and sensitivity of neurons to MPTP. Neurobiol Aging 2009; 30: 2021-30.

[49] Sonntag WE, Carter CS, Ikeno Y, et al. Adult-onset growth hormone and insulin-like growth factor I deficiency reduces neoplastic disease, modifies age-related pathology, and increases life span. Endocrinology 2005; 146: 2920-32.

[50] Lichtenwalner RJ, Forbes ME, Sonntag WE, Riddle DR. Adultonset deficiency in growth hormone and insulin-like growth factorI decreases survival of dentate granule neurons: insights into the regulation of adult hippocampal neurogenesis. J Neurosci Res 2006; 83: 199-210.

[51] Sonntag WE, Bennett $\mathrm{C}$, Ingram $\mathrm{R}$, et al. Growth hormone and IGF-I modulate local cerebral glucose utilization and ATP levels in a model of adult-onset growth hormone deficiency. Am J Physiol Endocrinol Metab 2006; 291: E604-10. 